\title{
Vascular Tumors of the Neck in Adults: 10-Year Experience in a Tertiary Center
}

\author{
Onur S. Goksel, MD, ${ }^{1}$ Emre Gok, MD,${ }^{1}$ Celalettin Karatepe, $\mathrm{MD},{ }^{2}$ Cagla Canbay Sarilar, MD,${ }^{1}$ \\ Mehmet Akif Onalan, MD, ${ }^{1}$ Metin Beyaz, MD, ${ }^{1}$ Ufuk Alpagut, $\mathrm{MD}^{1}$ \\ ${ }^{1}$ Istanbul University, Istanbul Medical Faculty, Cardiovascular Surgery Department, Istanbul, Turkey; ${ }^{2}$ Mustafa Kemal University, \\ Cardiovascular Surgery Department, Antakya
}

\section{ABSTRACT}

Introduction: The diagnosis and management of vascular lesions of the neck is a challenging task that requires a multidisciplinary approach. This retrospective study assesses the single center experience of vascular tumors of the neck.

Materials and methods: Patients diagnosed with a vascular tumor and/or a mass in close proximity to the carotid artery were identified from our records over a 10-year period. The demographic characteristics, clinical features, surgical approach, and outcomes were reviewed.

Results: Surgical excision of 17 vascular lesions were performed in 16 patients with a mean age of $51.56 \pm 17.35$ years at the time of operation. Intra- and/or postoperative clinical and histological assessment revealed unilateral glomus caroticum $(\mathrm{N}=11)$, glomus vagale $(\mathrm{N}=2)$, bilateral glomus caroticum $(\mathrm{N}=1)$, cavernous hemangioma $(\mathrm{N}=1)$, and carotid sheath tumor $(\mathrm{N}=1)$. In three patients, internal carotid artery, common carotid artery and vagal nerve were sacrificed to facilitate complete tumor excision. During the follow-up period, no tumor recurrences were observed, and the morbidity and mortality were minimal.

Conclusion: Preoperative evaluation concerning the size, extent, and anatomical relationships of the tumor thoroughly should be investigated. Multidisciplinary approach involving vascular surgery, otolaryngology, and radiology is preferred to treat these patients for better outcomes. Preoperative embolization in selected cases may decrease estimated blood loss and operative time.

\section{INTRODUCTION}

Vascular lesions of the head and neck region are complex and diverse. Carotid body tumors (CBTs), also called paragangliomas, are slow growing, painless neck masses originating from neural crest cells of the carotid arteries [Kruger 2010]. Other lesions vary from glomus vagale tumors, hemangiomas, venous malformations, and lymphatic malformations to arteriovenous malformations and carotid sheath tumors. In 1971, Shamblin et al proposed a classification system for

Received October 14, 2019; accepted Fanuary 15, 2020.

Correspondence: Dr. Onur S. Goksel, Istanbul Medical Faculty, Millet Cd. ÇAPA, Istanbul-Turkey; +90-212-414-20-00; fax: +90-216-348-02-69 (e-mail: onurgoksel@hotmail.com).
CBTs, based on the surgical resectability of these tumors and the relationship between the mass and the carotid artery wall. Since then, this classification widely has been used to describe the CBTs [Shamblin 1971] as well as guide surgical management and predict postoperative outcomes. Moreover, radiologists tend to classify neck lesion, according to the flow characteristics based on duplex ultrasound and magnetic resonance imaging (MRI) findings, which yield improved preoperative management and reduced perioperative complication rates [Griauzde 2015; Arya 2008].

The preferred method of treatment is surgery for most of the tumors, but it can be challenging due to the high vascularity of the tumor and close anatomical localization to cranial nerves. Therefore, diagnosis and management of these rare clinical entities remain cumbersome for the surgeon. We hereby present our decade-long experience with vascular tumors of the neck.

\section{MATERIALS AND METHODS}

Sixteen patients (mean age $51.56 \pm 17.35$ years, range between 17-61 years) diagnosed with a vascular tumor and/or a mass in close proximity to the carotid artery were referred to our university hospital clinic between 2007 and 2017. Hospital records, including preoperative evaluation and diagnostic imaging tests (such as duplex ultrasound, MRI, and computed tomography) as well as early- and midterm follow-up notes, were reviewed upon Institutional Scientific Committee approval. An informed consent for the procedures and study was obtained from each patient. After assessing the size, extent, and anatomic relationship of the tumor with both CT angiography and contrast-enhanced MRI, our standard approach was diagnostic angiography of the mass to yield its possible arterial supply from carotid arteries. Microcoilembolization of the feeder artery was performed in all cases except one within 48 hours before surgery (Figure 1A-C). After routine preoperative evaluation, all patients were operated under general anesthesia with endotracheal intubation. Surgery was indicated in all patients once diagnosed irrespective of the presence of symptoms of mass effect, such as dyspnea or dysphagia. The surgical technique was precise anatomic dissection and vascular control before complete tumor excision. For Shamblin I and II lesions, the tumor was dissected from the common carotid artery and its branches in the subadventitial plane. Blood flow was never interrupted in almost all of these patients, during the operation. Carotid 
Patient demographics, tumor classification and management

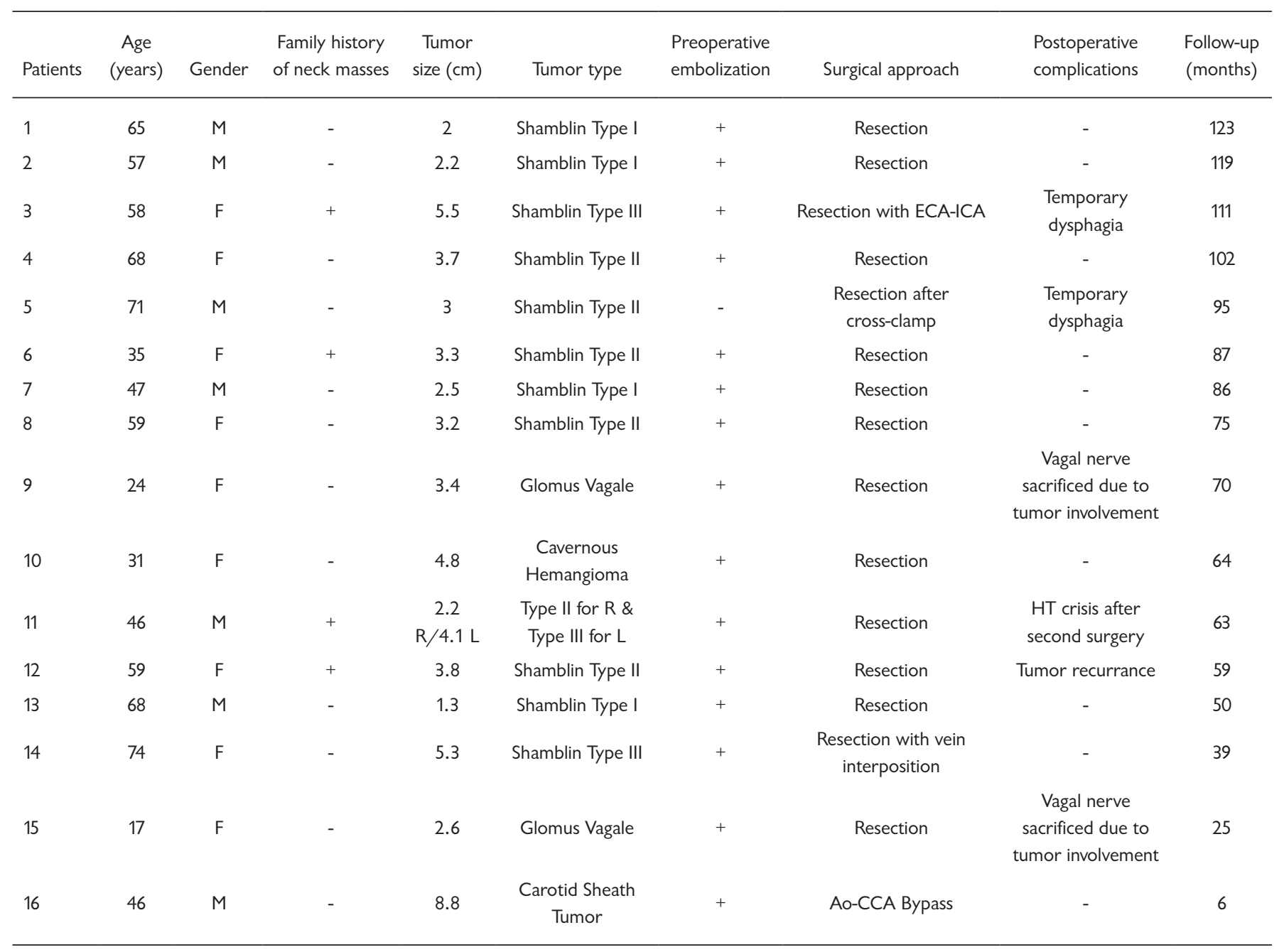

CCA, Common Carotid Artery; ECA, External Carotid Artery; ICA, Internal Carotid Artery; Ao, Aorta; HT, Hypertension

clamping was necessary in only one patient (Patient 5) in whom coil embolization preoperatively was not performed. Due to the high vascularity of these tumors, we placed cross clamp on the artery, resected the tumoral mass, and performed arteriotomy for shunting in this particular patient.

In one of the Shamblin type III cases, before the resection of the tumor, common carotid artery (CCA), internal (ICA), and external carotid arteries (ECA) were controlled with tape. As a general approach, subadventitial dissection was undertaken in the caudocranial direction. Following separation of the tumor from ICA and ECA were partially excised along with the mass, leaving $0.5 \mathrm{~cm}$ wide healthy bordering tissue. After the excision of the mass, the external carotid artery was anastomosed distally to the internal carotid artery. Complete tumor resection was not possible without the sacrifice of the internal carotid artery in each patient with a Shamblin type III tumor. An autologous saphenous vein graft was used for an interposition procedure between common carotid artery and distal internal carotid artery. Except for these particular patients, complete tumor resection also was possible in two patients with glomus vagale tumor with no the need of an additional vascular procedure, but sacrifice vagal nerve for thorough tumor excision.

All survivors conformed to the follow-up protocol. Thorough medical examination was performed and recorded, during each outpatient visit at every three months up until 24 months. All patients had control contrast-enhanced computerized axial tomography (CAT) scan at the postoperative third month and then annually, if asymptomatic.

The statistical analysis was performed using the SPSS version 20.0 software program (SPSS Inc., Chicago, IL). The data was presented as percentages or mean \pm standard deviation (SD). Univariate comparisons were computed using a chi-square test or Fisher's exact test for categorical variables and $t$ tests for continuous variables. Any factor with a $P$ value of $<.1$ on the univariate analysis was then entered into a multiple logistic regression analysis. A $P$-value of $<.05$ was considered to be significant. 


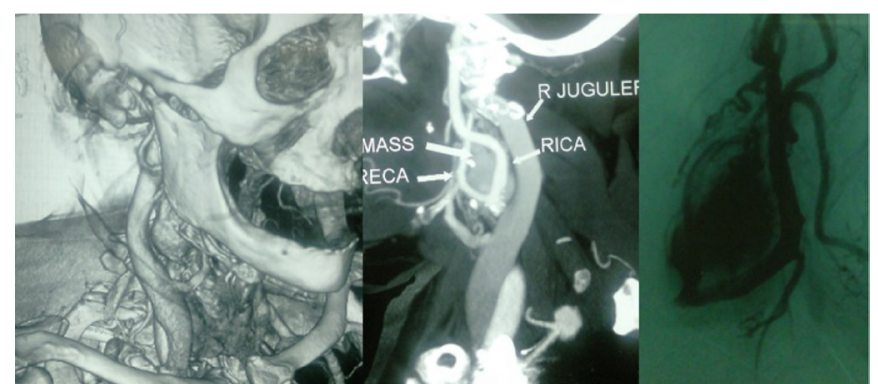

Figure 1. Glomus Carticum Tumor Type I. A, 3D reconstruction of the tumor; B, CT image of the tumor; C, Note the hypervascular structure of the tumor before embolization procedure.

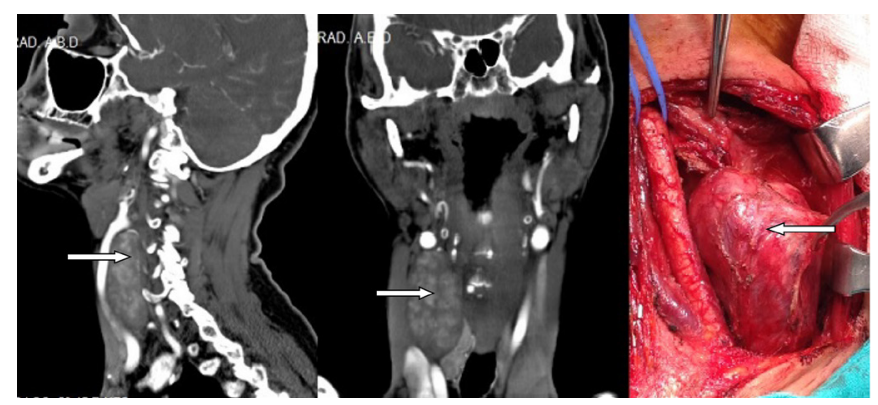

Figure 2. Glomus Caroticum Tumor Type II.

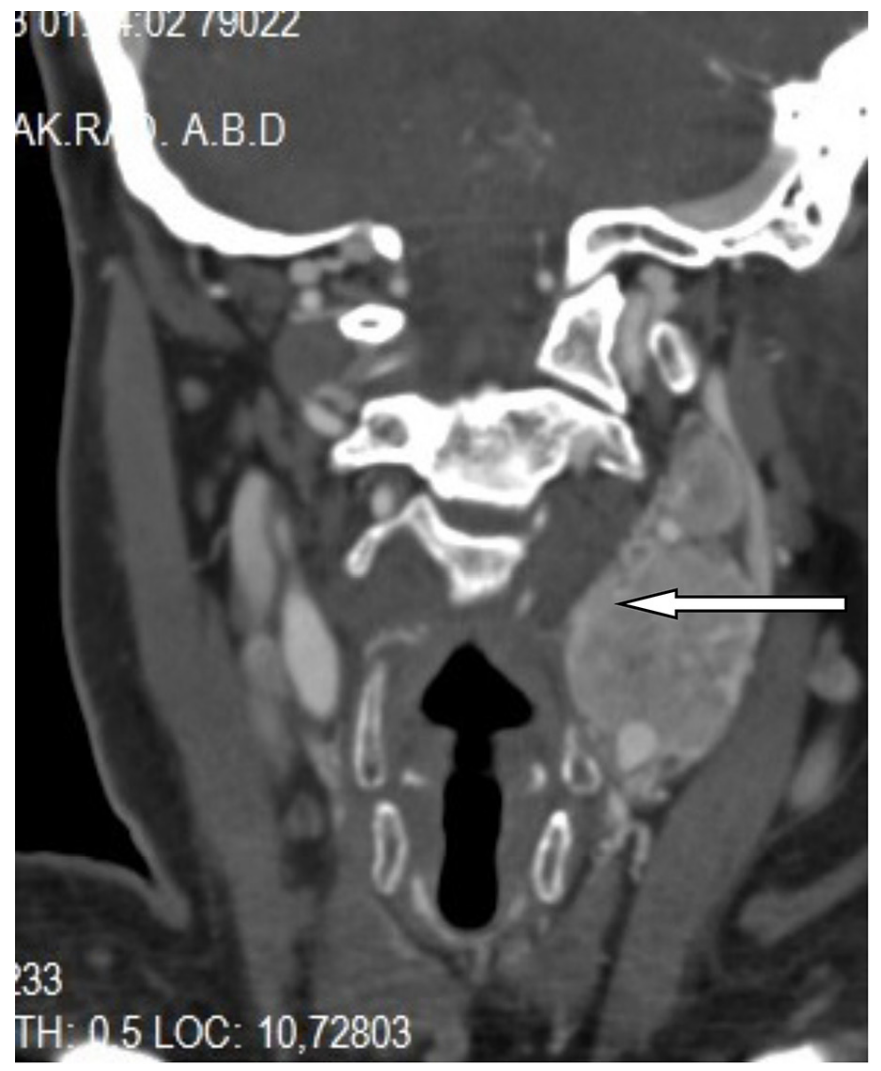

Figure 3. Glomus Caroticum Tumor Type III.

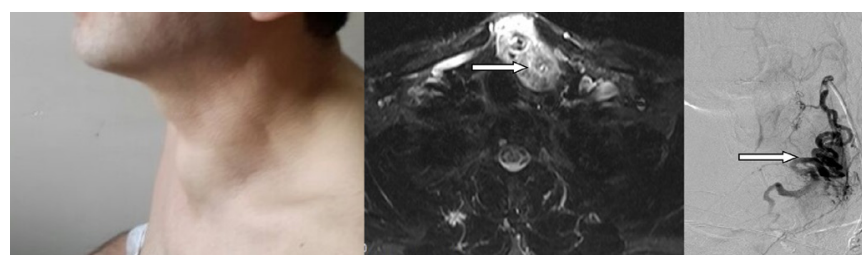

Figure 4. Cavernous Hemangioma.

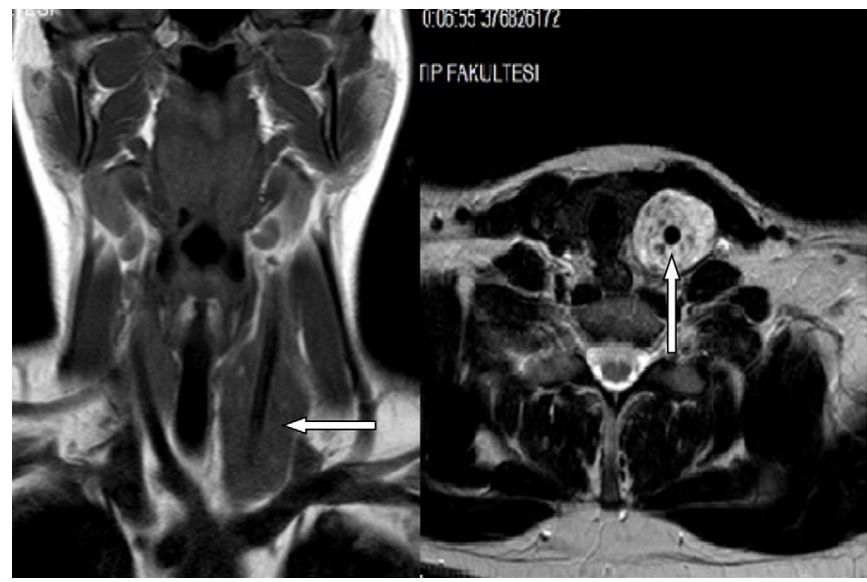

Figure 5. Carotid Sheath Tumor.

\section{RESULTS}

As the final diagnosis, 11 patients had glomus caroticum tumor, two patients had glomus vagale tumor, and one had bilateral caroticum tumors. One patient had cavernous hemangioma; another patient had a carotid sheath tumor. The female to male ratio was $1.28: 1$ with a mean age of $51.56 \pm$ 17.35 years at the time of operation. The follow-up period for the patients was 6 to 123 months. Patient characteristics are summarized in Table 1. One patient also was referred to our clinic with a mass of vascular origin in close proximity to the carotid artery, but she was excluded from our study because pathological microscopy of the extirpated mass yielded to a diagnosis of ectopic thyroid gland. We observed that in our patient population, patients with glomus vagale tumor $(\mathrm{N}=2)$ were significantly younger than rest of the group (17 and 24 years old, $P<.05$ with t-test).

Patients with glomus tumors (both caroticum and vagale types) predominantly were females (eight females vs. five males). Four patients with glomus caroticum tumor had a family history of similar neck masses. Out of 11 glomus caroticum cases, four patients were diagnosed with Shamblin type I tumor (Figure 2A-C), five with type II, and two with type III (Figure 3). On the other hand, the patient with bilateral glomus caroticum tumor was diagnosed with Shamblin type II for the right side and type III for the left side.

For the bilateral glomus caroticum case, we followed a two-step surgical approach. Considering the function of cranial nerves, we preferred to excise the smaller tumor first where the risk of cranial nerve damage was lower. In a 
separate session six months later, we resected the contralateral larger tumor, where the risk of neural damage was higher. The first surgery was performed without any resultant cranial nerve palsy. However, after the second surgery the patient experienced severe fluctuations in blood pressure during hospitalization and needed to be treated with sodium nitroprusside and central alpha-agonists to stabilize his blood pressure.

In contrast to CBTs, a transverse cervical incision was used for the case of cavernous hemangioma (Figure 4 A-C). After accessing the carotid sheath, the mass was dissected from the carotid artery and internal jugular vein. The vagus nerve was anatomically unrelated to the mass and preserved. Histopathological evaluation of the excised specimen revealed large cavernous vascular structures and supported the diagnosis as sclerosing cavernous hemangioma.

A patient with a carotid sheath tumor extending along the left CCA from its origin from aortic arch was operated through a partial upper sternotomy incision extending cranially anterior to left sternocleidomastoid muscle (Figure 5). Complete resection of the tumor encircling the left CCA with a bypass from the aortic arch to carotid bifurcation with an 8 mm- PTFE graft.

There was no perioperative mortality and pathologically, metastatic features were not detected in any of the patients. Tumor recurrence or any additional neurogenic dysfunction was not observed during the follow-up period.

\section{DISCUSSION}

Vascular tumors of the neck region, although rare, can often present with a diagnostic dilemma, particularly when they are asymptomatic or only mildly symptomatic besides paragangliomas. The architecture and vascularity of these lesions are usual keys to diagnosis. High-flow lesions (hemangiomas) readily can be distinguished from low-flow lesions (venous malformations and lymphatic malformations) with USG techniques, without the need for conventional angiography in the majority of cases. Paragangliomas, on the other hand, are cervical neoplasms of neuroectodermal paraganglion cells more commonly seen in the middle-aged female [Pellitieri 2004]. They can derive from carotid artery baroreceptors (glomus caroticum), internal jugular vein (glomus jugulare), vagal nerve (glomus vagale), or tympanic cavity (glomus tympanicum). Hormonally active paragangliomas are very rare [Kruger 2010]. None of our patients had functionally active tumors. In most cases, they presented with either a painless neck mass or randomly detected neck mass on imaging obtained for another reason.

Complete surgical resection is the treatment of choice for CBTs. The resection of tumors is performed via the transcervical approach by controlling proximal and distal segments of carotid arteries with specific attention to prevent cranial nerve injuries [Offergeld 2012; Wang 2000; Ozen 2015].

Due to the high vascularity of these tumors, preoperative embolization should be evaluated. Super-selective catheterization of the supplying branches for trans-arterial embolization with particulated agents or direct intra-tumoral injection with a permanent liquid polymerizing agent was used for this purpose [Casasco 1994]. Although direct intra-tumoral injection of glue has promising results and is helpful for better reduction of tumor vascularity, due to the risk of distal embolization of these agents, hemorrhagic risks from direct puncture and jugular vein migration, it has not widely been adopted. Because of these reasons, we performed preoperative embolization within 48 hours of surgery to all patients in our series except one case. However, these tumors might have numerous feeding arteries, reducing the effect of embolization, and represent a potential risk of cerebral infarction by embolic particles.

In Shamblin type II or III glomus caroticum patients, the risk of injury to carotid vessels and cranial nerve was approximately $40 \%$ to $50 \%$, due to the surgery since larger tumors with close proximity to critical structures could require a more complex surgery [Shamblin 1971]. With the improvements in modern vascular surgical techniques and sophisticated diagnostic modalities, complication rates decreased to about 25\% [O’Neill 2011]. In our series, we observed two patients in Shamblin type III, and only one received saphenous vein bypass to the internal carotid artery after successful resection of the tumor $(50 \%)$. Cranial nerve damage was observed in three of the 11 CBT patients (27\%), including the above-mentioned glomus vagale and Shamblin type III glomus caroticum patients.

Similar to the surgical treatment of CBTs, Miller et al suggest the surgical resection of vagal paragangliomas [Miller 2000]. These tumors can reach to the base of the skull, so approaches that are more radical might be necessary. However, it is almost impossible to resect these tumors without intraoperatively sacrificing the vagal nerve; therefore, nerve deficit rates are higher for the resection vagal paragangliomas compared with CBTs, which increases morbidity and mortality. Due to the aforementioned dire complications, preoperative embolization of glomus vagale tumor is still controversial. Patient selection and life expectancy is critical, due to the possibility of incurring neurological sequelae [Pellitieri 2004].

In patients with bilateral tumors, the surgical procedures should not be done simultaneously to both sides because of potential vascular or cranial nerve injury. It is predictable that the risk of intraoperative cranial nerve injury observed in bilateral and/or large lesions is higher so it is essential to evaluate the preoperative status of cranial nerves (VII, X, XI, XII) before the second intervention. In addition, after the second operation, surgeons should be aware of possible suppression of the two carotid sinuses and the risk of hypertensive instability. De Toma et al described this baroreflex failure syndrome and evaluated baroreceptor sensitivity in their report. Authors suggested that this phenomenon might be related to the denervation of the carotid sinus and the baroreceptor arc reflex [De Toma 2000]. In our experience, our patient was asymptomatic before the second operation, but after the second session, the patient developed a serious hypertensive crisis unresponsive to first line antihypertensive therapies. Only one patient in our series had bilateral glomus caroticum tumors and was operated sequentially in a 6-month period. No patients experienced ipsilateral tumor recurrence 
in the midterm, which is significantly lower than $4.9 \%$ rate of recurrence as reported by other authors [Pellitieri 2004; Patetsios 2002].

Carotid sheath tumors are very rare tumors and can be of neural origin. When derived from the cervical sympathetic chain, they usually present as asymptomatic masses located in the posterior cervical regions, at paravertebral level. In our patient, the sheath tumor also originated from the sympathetic nerve located in the carotid sheath, extending to the mediastinum, and the vagus nerve was completely penetrated by the tumor. Its differentiation from CBTs is difficult clinically and several authors have recommended the use of radiologic scans. The main imaging criterion to differentiate a schwannoma from a CBT is the lack of hypervascularity [Panneton 2000]. Compared with CBTs, a schwannoma can easily be peeled away from the carotid or jugular vessels unless invasive in nature. In our patient, we performed en-bloc carotid resection and PTFE grafting, due to the intraoperative suspicion of malignancy. Hemangiomas are benign vascular tumors composed of large dilated blood vessels and containing large blood-filled spaces. The majority of them are located in the head and neck region, such as the tongue, parotid gland, and tympanic membrane, within the sternocleidomastoid muscle or carotid sheath [Waldman 2006; Zagzag 2009]. They do not regress, and the treatment is surgical intervention. Our experience was similar to a previous report, and the tumor was completely removed [Zagzag 2009].

It is of note that surgery was indicated in all patients once diagnosed irrespective of the presence of symptoms of mass effect, such as dyspnea or dysphagia, in accordance with the idea that the surgical excision may be more difficult as the tumor size increases due to local invasion and due to the possible risk of metastases in time. A thorough evaluation of the mass with its surrounding structures using CT angiography in addition to MRI is utmost important for diagnosis, extension, and careful planning as well as conventional angiography for determination of vascularity and coil-embolization.

\section{CONCLUSION}

A careful preoperative evaluation of tumor characteristics concerning its size, extent and relationship with adjacent vessels and nerves should be obtained. A multidisciplinary approach involving vascular surgery, otolaryngology, and radiology should be used to treat these patients for better outcomes. Early diagnosis of vascular neck tumors allows timely treatment and minimizes the risk of cranial nerve and vessel injury.

\section{REFERENCES}

Arya S, Rao V, Juvekar S, Dcruz AK. 2008. Carotid body tumors: objective criteria to predict the Shamblin group on MR imaging. AJNR Am J Neuroradiol 29(7):1349-54.

Casasco A, Herbreteau D, Houdart E, et al. 1994. Devascularization of craniofacial tumors by percutaneous tumor puncture. AJNR Am J Neuroradiol 15:1233e9.

De Toma G, Nicolanti V, Plocco M, et al. 2000. Baroreflex failure syndrome after bilateral excision of carotid body tumors: an underestimated problem. J Vasc Surg 31:806-10.

Griauzde J, Srinivasan A. 2015. Imaging of vascular lesions of the head and neck. Radiol Clin North Am. 53:197-213.

Kruger AJ, Walker PJ, Foster WJ et al. 2010. Important observations made managing carotid body tumors during a 25 -year experience. J Vasc Surg 52:1518-1523.

Miller RB, Boon MS, Atkins JP, et al. 2000. Vagal paraganglioma: the Jefferson experience. Otolaryngol Head Neck Surg 122:482-487.

Offergeld C, Brase C, Yaremchuk S, et al. 2012. Head and neck paragangliomas: clinical and molecular genetic classification. Clinics (Sao Paulo) 67(Suppl 1): 19-28.

O’Neill S, O'Donnell M, Harkin D et al. 2011. A 22- year Northern Irish experience of carotid body tumors. Ulster Med J. 80(3): 133-140.

Ozen A, Unal EU, Kubat E, et al. 2015. Diagnosis and Surgical Treatment of Carotid Body Tumors. J Clin Anal Med 6(4):490-2.

Panneton JM, Rusnak BW. 2000. Cervical sympathetic chain schwannomas masquerading as carotid body tumours. Ann Vasc Surg 14:519-24.

Patetsios P, Gable DR, Garret WV, et al. 2002. Management of carotid body paragangliomas and review of 30-year experience. Ann Vasc Surg $16: 331-338$

Pellitieri PK, Rinaldo A, Myssiorek D et al. 2004. Paraganglioma of the neck. Oral Oncology 40:563-75.

Shamblin WR, ReMine WH, Sheps SG, et al. 1971. Carotid body tumor (chemodectoma). Clinicopathologic analysis of ninety cases. Am J Surg. 122(6):732-9.

Waldman EH, Goldenberg D, Califano J, et al. 2006.Venous malformation of the sternomastoid muscle. Otolaryngol Head Neck Surg 135:325-327.

Zagzag J, Morris LG, DeLacure MD. 2009. Cavernous hemangioma of the carotid sheath. Otolaryngol Head Neck Surg. 140(4):608-9. 